\title{
Exploring the Geographic Variation of Pediatric Renovascular Hypertension Etiology Through the Lens of Evolutionary Medicine: A Perspective
}

\author{
Jaineet Singh Chhabra, MS ${ }^{1}$ \\ ${ }^{1}$ Marshall University School of Medicine. 1600 Medical Center Dr, Huntington, WV 25701
}

June 22, 2021

Keywords: Pediatric, Renovascular, Hypertension, Evolution, Medicine

Abbreviations: renal artery stenosis (RAS), fibromuscular dysplasia (FMD), Takayasu arteritis (TA), ACE insertion/deletion (ACE I/D), renovascular hypertension (RVH), angiotensin I (ang I), angiotensin II (ang II), angiotensin converting enzyme (ACE), midaortic syndrome (MAS), angiotensinogen (AGT)

Correspondence:

$113014^{\text {th }}$ Street, Huntington, WV, 25701, Apartment 133

Email: chhabra@marshall.edu

\begin{abstract}
Though pediatric renal artery stenosis (RAS) is the most common cause of secondary hypertension in children, it is uncommon. Interestingly, geographic variance of disease etiology has been reported in the literature. Fibromuscular dysplasia (FMD) contributes to most pediatric RAS cases in Europe and North America while Takayasu arteritis (TA) is the main cause in Asia. The high prevalence of essential hypertension observed in African Americans has been well-documented, in which there may be latitude dependent genes that once protected ancestors from dehydration but became maladaptive in modern, salt heavy environments. However, a review of the literature yielded little information that could support a latitudinal association with the aforementioned geographic variance concerning the different etiologies contributing to pediatric RAS. Upon further exploration, the ACE insertion/deletion (ACE I/D) polymorphism surfaced as a possible link between FMD and TA when considering renovascular disease in children. The ACE I allele is associated with greater endurance in whites while the D allele may provide this advantage to Asians, suggesting that endurance and increased susceptibility to pediatric RAS may be involved an evolutionary tradeoff. This preliminary discussion may eventually guide future research efforts concerning identification of gene patterns and perhaps lead to development of earlier treatment.
\end{abstract}

\section{Article}

Though vascular disease in children is a growing concern due to sedentary lifestyle, poor diet and obesity, it remains a rarity. Small size, continued development of the body, and vascular conduit durability pose unique challenges regarding treatment of pediatric vascular conditions. These include trauma, tumor invasion of major abdominal vessels, aortoiliac aneurysm, acute or chronic arterial occlusion, and renovascular hypertension. ${ }^{2}$ From an evolutionary medicine perspective, recognizing rare diseases that are unique to particular populations or subpopulations can spark interesting discussions that may elucidate otherwise hidden risk factors and maximize timely diagnosis. Pediatric renovascular hypertension might be one such condition. 
When considering pediatric hypertension, one may question if there are any unique contributors pertinent to a younger population susceptible to developing high blood pressure. The most common cause of secondary hypertension is renal artery stenosis (RAS). As opposed to atherosclerosis in adults, etiologies of pediatric RAS appear to vary by geography and disease process; fibromuscular dysplasia (FMD) contributes to most cases in North America and Europe while Takayasu arteritis (TA) prompts renovascular lesions largely in Asian and South African youth. ${ }^{3}$ FMD is a rare, idiopathic vascular disease that causes non-inflammatory, nonatherosclerotic disease mostly in renal and carotid arteries; it appears to be most common in Caucasians and women. ${ }^{4,5} \mathrm{TA}$ is a granulomatous large vessel vasculitis involving mostly the aorta and its proximal branches, affecting mostly young, Asian females. ${ }^{6}$ In approximately $10 \%$ of hypertensive children, renovascular lesions prompt increased renin secretion, eventual systemic hypertension, and potentially myocardial infarction or stroke should treatment be delayed or ineffective. ${ }^{3,7}$ Discussing why there is geographic variation of disease processes causing pediatric RAS may yield important points for future investigators interested in developing preventative or targeted therapies.

A general breakdown of the proximate mechanisms contributing to RAS provides some necessary background for the forthcoming discussion of potential ultimate causes. First described by Goldblatt et al., ${ }^{8}$ the pathophysiology of renovascular hypertension (RVH) consists of several mechanisms that arise subsequent to ischemia of the kidneys. Secretion of renin by the juxtaglomerular apparatus is triggered by either renal baroreceptors responsible for detecting reduced perfusion, macula densa sensing of low sodium levels, or activation of beta-adrenergic receptors. Renin is then secreted into the blood and cleaves angiotensinogen produced by the liver into angiotensin I (ang I) which is transformed into angiotensin II (ang II) by angiotensin converting enzyme (ACE) produced by the lung and kidney. Ang II induced vasoconstriction of vascular smooth muscle, aldosterone release, and fibrotic vessel wall thickening all contribute, if prolonged, to eventual hypertensive injury which may result in decreased blood flow. ${ }^{9}$ In addition to isolated RAS, RVH can be concomitantly or separately caused by midaortic syndrome (MAS). MAS is characterized by segmental narrowing of the distal descending thoracic or abdominal aorta, in which concomitant RAS is observed in $60 \%$ of these children. ${ }^{10}$

The discussion now takes on a more evolutionary tone. Though the etiologies of RAS/RVH vary with age and are perhaps narrower in scope compared with essential hypertension overall, attempting to understand the increased hypertension prevalence observed in African American adults and other populations can yield valuable insights regarding the impact geography can have on hypertension etiology. One proposed hypothesis associated with this topic involves natural selection for sodium-conserving genotypes that protected ancestral populations from dehydration in hot climates. Some studies have identified several allelic variants that contribute to heat adaptation via sodium conservation, such as the $-6 \mathrm{~A}$ allele of the promoter of the angiotensinogen (AGT) gene, the $825 \mathrm{~T}$ allele of the GNB3 gene, the $-946 \mathrm{G}$ allele of the epithelial sodium channel $\alpha$-subunit gene, and the $47 \mathrm{~A}$ and $79 \mathrm{C}$ alleles of the $\beta 2$-adrenergic receptor gene. These ancestral variants exhibit latitude dependency, in which they are much more prevalent in African populations residing near the equator and less so in areas farther away from the imaginary planetary line. ${ }^{11}$ In extreme temperatures sodium conservation was advantageous given that increased fluid retention slowed dehydration. However, salt-rich environments, such as those present in the United States, have negatively affected descendants given that retention becomes maladaptive in lower temperatures. This latitude-dependent phenomenon may even occur in lower risk groups, such as Asians. Nguyen et al. found, despite low mean body mass indices, in their study that the prevalence of hypertension in Chinese, Indonesian, and Vietnamese men were 22.9\%, $24.8 \%$, and $14.4 \%$ and $16.6 \%, 26.9 \%$, and $11.7 \%$ in women respectively. ${ }^{12}$ It is important to note, however, that a subsequent literature search revealed little data supporting a latitude dependency that could explain the geographic variation associated with pediatric RAS.

No causative genes or distinct pathogenic factors associated with FMD have been determined to date. ${ }^{13}$ However, a study conducted by Bofinger et al. found that the ACE insertion/deletion (ACE I/D) polymorphism presents at a higher frequency in renal FMD patients compared with controls. The ACE I allele correlates with lower circulating ACE levels and potentially lower ang I levels. Given that ang II modulates growth of smooth muscle, ACE I may increase susceptibility of arterial media remodeling and subsequent 
development of multifocal renal arterial FMD. This differs from the arterial intima thickening observed in atherosclerotic RAS which is associated with the ACE D allele. ${ }^{14}$ Interestingly, an earlier study conducted by Bloem et al. found in their cohort that white children and adolescents homozygous for D alleles exhibit a higher level of ACE activity compared with the D/I genotype or those homozygous for I alleles. ${ }^{15}$ It should be noted that this study did not disclose any subjects with FMD, suggesting that whites homozygous for ACE I might have a lower risk of essential hypertension but increased risk of FMD-induced hypertension which manifests at a young age. If this is confirmed by future studies, could there be a tradeoff benefit in a subset of white ancestors that increased FMD susceptibility in descendants? The literature reports that increased endurance is associated with lower resting blood pressure. ${ }^{16-18}$ Though understanding the association between the ACE I/D polymorphism and race is complex and largely inconclusive, it has been well-documented that the ACE I allele is associated with increased endurance in Europeans. ${ }^{19-22}$ It has been proposed that endurance may have played a role in helping hominin scavengers outcompete other terrestrial vertebrate scavengers for carcasses. ${ }^{22}$ Setting chance aside for sake of exploration, it appears plausible that possessing the ACE I allele maximized endurance for scavengers but in turn predisposed some descendants to increased FMD susceptibility. However, it is unclear as to why a benefit that perhaps emerged as early as 4.4 million years ago poses this particular disadvantage mostly in whites today.

Shifting focus to the connection between TA and pediatric RAS in Asians, a meta-analysis by Lee et al. found an association between the ACE I/D polymorphism and susceptibility to several forms of vasculitis, including Bechet Disease and Henoch-Schonlein purpura. ${ }^{23}$ Though they only compiled 12 studies and did not discuss TA, their findings suggest that there might be a common pathway involved in FMD and TA manifestation. In contrast to the European population, several studies have found that ACE I/D polymorphisms in the Japanese exhibit an opposite pattern regarding endurance. ${ }^{24,25,26}$ The ID and DD genotype of the ACE I/D polymorphism in the Japanese population is associated with greater endurance. The etiology of TA is not well understood, but the strongest connection concerns the Japanese population and the HLA-B52 serotype. It is important to note that this serotype is present in some other populations. However, it appears that Japanese who are HLA-B52 positive carry the worst prognosis. ${ }^{27}$ Given the lack of understanding of the genes responsible for TA manifestation, it would perhaps be useful for studies to clarify whether any association exists between the ACE I/D polymorphism and HLA-B52 serotype in TA patients. Might the aforementioned scavenging benefit apply here as well, in which a different pattern of genes be responsible for increased TA, as opposed to FMD, susceptibility in the Japanese population?

Though it is currently unclear what fitness advantage greater endurance offered ancestors of each discussed population, possessing the ACE-I allele is a trait that may be part of an evolutionary tradeoff where the benefit of endurance is offset by increased risk of FMD development or TA. If both groups required greater endurance to scavenge, why were a different pattern of genes required? Could this be due to environmental differences that shaped two different diseases processes involved in a similar tradeoff? It is debatable whether questions like these will yield clinically relevant answers. However, should the geographic variation associated with FMD or TA-mediated RAS in the pediatric population not be due to chance, further discussion of potential ultimate causes may eventually lead to studies dedicated to identifying the gene patterns responsible for this observation and perhaps lead to earlier treatment.

\section{References}

1. Short KR, Blackett PR, Gardner AW, Copeland KC. Vascular health in children and adolescents: effects of obesity and diabetes. Vasc Health Risk Manag . 2009;5:973-990. doi:10.2147/vhrm.s7116

2. Min SK, Cho S, Kim HY, Kim SJ. Pediatric Vascular Surgery Review with a 30-Year-Experience in a Tertiary Referral Center. Vasc Specialist Int . 2017;33(2):47-54. doi:10.5758/vsi.2017.33.2.47

3. Villegas L, Cahill AM, Meyers K. Pediatric Renovascular Hypertension: Manifestations and Management. Indian Pediatr. 2020;57(5):443-451.

4. Baradhi KM, Bream P. Fibromuscular Dysplasia. [Updated 2020 Jul 12]. In: StatPearls [Internet]. Treasure Island (FL): StatPearls Publishing; 2021 Jan-. Available from: https://www.ncbi.nlm.nih.gov/books/NBK493204/ 
5. Onen F, Akkoc N. Epidemiology of Takayasu arteritis. Presse Med . 2017;46(7-8 Pt 2):e197-e203. doi:10.1016/j.lpm.2017.05.034

6. Rana MN, Al-Kindi SG. Prevalence and manifestations of diagnosed fibromuscular dysplasia by sex and race: Analysis of $>4500$ FMD cases in the United States. Heart Lung . 2021;50(1):168-173. doi:10.1016/j.hrtlng.2020.09.022

7. St Peter SD, Ostlie DJ. A review of vascular surgery in the pediatric population. Pediatr Surg Int . 2007; 23(1): 1-10.

8. Harry Goldblatt, James Lynch, Ramon F. Hanzal, Ward W. Summerville; STUDIES ON EXPERIMENTAL HYPERTENSION: I. THE PRODUCTION OF PERSISTENT ELEVATION OF SYSTOLIC BLOOD PRESSURE BY MEANS OF RENAL ISCHEMIA. J Exp Med 1 March 1934; 59 (3): 347-379. doi: https://doi.org/10.1084/jem.59.3.347

9. Nair R, Vaqar S. Renovascular Hypertension. [Updated 2020 Dec 2]. In: StatPearls [Internet]. Treasure Island (FL): StatPearls Publishing; 2021 Jan-. Available from: https://www.ncbi.nlm.nih.gov/books/NBK551587/

10. Patel, P.A., Cahill, A.M. Renovascular hypertension in children. CVIR Endovasc 4, 10 (2021). https://doi.org/10.1186/s42155-020-00176-5

11. A.B. Weder, Evolution and hypertension. Hypertension, 49 (2007), pp. 260-265

12. Nguyen TT, Adair LS, Suchindran CM, He K, Popkin BM. The association between body mass index and hypertension is different between East and Southeast Asians. Am J Clin Nutr . 2009;89(6):19051912. doi:10.3945/ajcn.2008.26809

13. Olin JW, Gornik HL, Bacharach JM, Biller J, Fine L, Gray B, et al. Fibromuscular dysplasia: state of the science and critical unanswered questions: a scientific statement from the American Heart Association. Circulation . 2014;129(9):1048-1078. doi:10.1161/01.cir.0000442577.96802.8c

14. Bofinger A, Hawley C, Fisher P, Daunt N, Stowasser M, Gordon R. Polymorphisms of the reninangiotensin system in patients with multifocal renal arterial fibromuscular dysplasia. J Hum Hypertens . 2001;15(3):185-190. doi:10.1038/sj.jhh.1001144

15. Bloem LJ, Manatunga AK, Pratt JH. Racial difference in the relationship of an angiotensin Iconverting enzyme gene polymorphism to serum angiotensin I-converting enzyme activity. Hypertension . 1996;27(1):62-66. doi:10.1161/01.hyp.27.1.62

16. Cornelissen VA, Fagard RH. Effects of endurance training on blood pressure, blood pressureregulating mechanisms, and cardiovascular risk factors. Hypertension . 2005;46(4):667-675. doi:10.1161/01.HYP.0000184225.05629.51

17. Kelley GA, Kelley KA, Tran ZV. Aerobic exercise and resting blood pressure: a meta-analytic review of randomized, controlled trials. Prev Cardiol. 2001;4: 73-8

18. Whelton SP, Chin A, Xin X, He J. Effect of aerobic exercise on blood pressure: a meta-analysis of randomized controlled trials. Ann Intern Med. 2002; 136: 493-503.

19. Gayagay G, Yu B, Hambly B, Boston T, Hahn A, Celermajer D, et al. Elite endurance athletes and the ACE I allele-the role of genes in athletic performance. Hum Genet . 1998;103(1):48-50. doi:10.1007/s004390050781

20. Montgomery HE, Marshall R, Hemingway H, Myerson S, Clarkson P, Dollery C, et al. Human gene for physical performance. Nature 393: 221-222, 1998. doi:10.1038/30374.

21. Nazarov IB, Woods DR, Montgomery HE, Shneider OV, Kazakov VI, Tomilin NV, et al. The angiotensin converting enzyme I/D polymorphism in Russian athletes. Eur J Hum Genet 9: 797-801, 2001. doi:10.1038/sj.ejhg.5200711.

22. Ruxton GD, Wilkinson DM. Endurance running and its relevance to scavenging by early hominins. Evolution. 2013 Mar;67(3):861-7. doi: 10.1111/j.1558-5646.2012.01815.x. Epub 2012 Oct 25. PMID: 23461334.

23. Lee YH, Choi SJ, Ji JD, Song GG. Associations between the angiotensin-converting enzyme insertion/deletion polymorphism and susceptibility to vasculitis: a meta-analysis. J Renin Angiotensin Aldosterone Syst . 2012;13(1):196-201. doi:10.1177/1470320311434240

24. Kumagai H, Tobina T, Ichinoseki-Sekine N, Ryo K, Tsuzaki T, Zempo H, et al. Role of selected 
polymorphisms in determining muscle fiber composition in Japanese men and women. J Appl Physiol (1985) . 2018;124(5):1377-1384. doi:10.1152/japplphysiol.00953.2017

25. Tobina T, Michishita R, Yamasawa F, Zhang B, Sasaki H, Tanaka H, et al. Association between the angiotensin I-converting enzyme gene insertion/deletion polymorphism and endurance running speed in Japanese runners. J Physiol Sci 60: 325-330, 2010. doi:10.1007/s12576-010-0100-4

26. Wang G, Mikami E, Chiu LL, DE Perini A, Deason M, Fuku N, et al.Med Sci Sports Exerc 45: 892-900, 2013. doi:10.1249/MSS.0b013e31827c501f.

27. Russo RAG, Katsicas MM. Takayasu Arteritis. Front Pediatr . 2018;6:265. Published 2018 Sep 24. doi:10.3389/fped.2018.00265 